\title{
Navigating the science social mediaverse
}

\author{
Social media have emerged as a key communication tool for scientists. Here we explore how to reap the benefits \\ and avoid the pitfalls of social networking.
}

( ver the past decade, the reach of social networking has expanded steadily to satisfy the increasing appetite of the average internet user to consume online content and connect in different and more interesting ways. The social-networking revolution did not bypass the world of science, which has embraced social media for science communication and professional networking.

Nowadays there is a wealth of platforms for scientists to choose from, each providing overlapping but distinct functionalities. Among the most popular sites globally is Facebook, which offers the ability to compose lengthy, multimedia-rich posts and to create groups and pages dedicated to specific interests. Despite these attractive features, according to a 2014 Nature survey ${ }^{1}$, the majority of scientists regularly visiting this network did not use it professionally. In contrast, Twitter was the platform surveyed scientists used to post their work and discover and discuss research. The fact that this microblogging site encourages brevity by limiting posts to a maximum of 280 characters, but includes the ability to enrich them with links, photos and videos, might hold a certain appeal for scientists used to stripping complex information to the essentials. Facebook's sister site, Instagram, is also popular, and despite its more limited functionality, given its focus on images, it can also be a useful means for science communication and public engagement ${ }^{2}$. Mainland China, where these networks are not accessible, is nevertheless the biggest social-media market in the world, with domestic multipurpose super apps such as WeChat and Weibo engaging hundreds of millions to over a billion users monthly. The ubiquity of these apps for tasks ranging from private messaging and public social-media posts to digital payments makes them powerful, widely used tools for promoting, discovering and discussing scientific content. Beyond these publicly open and multifunctional social networks, scientists also interact through professionally-geared networks such as LinkedIn and academia-specific ones such as Academia.edu and ResearchGate.

A clear benefit of using social media is the wealth of science-related information that is literally at the user's fingertips. Nowadays the news media and the majority of research institutes, funding organizations and journals have active social-media accounts, often on multiple platforms. Moreover, many scientists post extensively about their work. The more Twitter-savvy researchers often compose 'tweetorials'-multi-tweet threads that provide deeper insight into a paper by circumventing the strict character limit. This has turned sites such as Twitter into 24/7 science news outlets that can help researchers stay abreast of the latest scientific developments. An added bonus is the ability to interact directly with each post, which allows the crowdsourced assessment and discussion of the latest discoveries, whether they are publicized through live-tweeted conference presentations, preprints or peer-reviewed papers. This aspect of digital engagement has proven invaluable throughout the COVID-19 pandemic, during which the rapid dissemination and evaluation of scientific findings has been essential to understanding the virus and disease, and to educating the general public and fighting misinformation. The participation of scientists in non-academic social-networking sites is also important for building trust by humanizing them in the eyes of the general public, who might otherwise not have been exposed to their science or personalities. Indeed, some scientists have gained hundreds of thousands of followers on Twitter through their prolific, trustworthy and informative posts.

Staying up to date with one's field and building a wider scientific network is also helpful for career development, both in terms of furthering one's own research by gaining knowledge, inspiration and collaborations, and by learning about open positions and funding opportunities. Scientists on the job market can publicize their accomplishments and reach out to prospective employers and funders through additional channels.

Crucially, social media are a means for expanding one's professional network beyond their immediate regional or discipline-specific scientific circle, something that can equalize networking opportunities for researchers who do not have the ability or resources to travel widely. The value of fostering a global scientific community online was evident during the past year of extended lockdowns and limited international travel. In recent years, social media have also been instrumental in increasing engagement with grassroots movements advocating for racial, gender and LGBTQ equality and inclusion.

This being the internet, things are not always rosy. The information deluge of social media makes productive engagement time-consuming, and for those who wish to be involved for professional reasons, it risks contributing to work-life imbalance. It can also lead to superficial regurgitation of information without in-depth assessment, which sometimes results in the dissemination of unsound or misinterpreted findings. Curating one's feed on the basis of specific interests and trusted sources and limiting access time can help one avoid constant scrolling to identify useful posts. Moreover, fact-checking information before sharing is essential, and this includes the source, as fake accounts and bots abound. On the flip side, such a perfectly curated feed can create an echo chamber that reinforces one's own views and limits exposure to diverse opinions, healthy debate and constructive criticism. The last is especially important when publicizing one's own achievements and findings. However, with social-media exposure comes vulnerability to online trolls, or the mere hostility and bad manners of some users over a difference of opinion. Whether and how to engage is up to each person; however, a way to protect oneself and others from problematic online behaviors is to mute, unfollow or block and report misbehaving users.

A separate issue with using social media in science is that conflicts of interest are hard to discern and are often not disclosed, as highlighted in a study of potential financial conflicts of interest among hematologistoncologists on Twitter ${ }^{3}$. Mentioning them in relevant posts and including them in one's user profile is a good way to encourage transparency. Similarly, ensuring that patient privacy is maintained during social-media discussions is essential.

Finally, although an active social-media presence may lead to useful interactions, collaborations and even friendships, oftentimes online connections can be without substance and, in the worst cases, can even be deceptive. Although extreme cases are hopefully rare, it is a good idea 
to be mindful of red flags while remaining open to new connections.

Social media, with their many pros and cons, have become an integral part of the digital transformation of our personal and professional lives. Many say that when venturing into the social networking world, it is important to develop and maintain a clear digital identity. How is that best achieved for scientists? Some opt to have a strictly professional profile online. Others happily share aspects of their personal lives with a global audience of fellow users.
Some prefer to remain 'lurkers' who consume content but rarely interact directly. Others engage in a limited manner, and yet others make considerable effort to develop their online persona and academic 'brand'. In reality, there is not a one-size-fits-all way to connect in digital spaces, and user choices of how to interact may change over time. Regardless of how individual scientists choose to use social media, the important thing is to keep digital engagements genuine, civilized and interesting.
Readers who are active on Twitter are welcome to follow our journal at $@$ NatureCancer to stay up to date on new content, special projects and news relevant to the cancer research field.

Published online: 23 June 2021

https://doi.org/10.1038/s43018-021-00233-5

References

1. Van Noorden, R. Nature 512, 126-129 (2014). 14 August.

2. Hines, H. \& Warring, S. Nature https://doi.org/10.1038/d41586019-00493-3 (2019).

3. Tao, D. L. et al. JAMA Intern. Med. 177, 425-427 (2017). 